SPhT-99/030

INLN-99/003

\title{
Condensation and Metastability in the 2D Potts Model
}

\author{
J. L. Meunier ${ }^{1, a}$ and A. Morel ${ }^{2, b}$ \\ May 16, 2018 \\ ${ }^{1}$ Institut non Linéaire de Nice-Sophia Antipolis, 1361 Route des Lucioles, \\ 06560 Valbonne, France \\ ${ }^{2}$ Service de Physique Théorique de Saclay, CE-Saclay, \\ F-91191 Gif-sur-Yvette Cedex, France
}

\begin{abstract}
For the first order transition of the Ising model below $T_{c}$, Isakov has proven that the free energy possesses an essential singularity in the applied field. Such a singularity in the control parameter, anticipated by condensation theory, is believed to be a generic feature of first order transitions, but too weak to be observable. We study these issues for the temperature driven transition of the $q$ states $2 D$ Potts model at $q>q_{c}=4$. Adapting the droplet model to this case, we relate its parameters to the critical properties at $q_{c}$ and confront the free energy to the many informations brought by previous works. The essential singularity predicted at the transition temperature leads to observable effects in numerical data. On a finite lattice, a metastability domain of temperatures is identified, which shrinks to zero in the thermodynamical limit.

${ }^{b}$ morel@spht.saclay.cea.fr
\end{abstract}

${ }^{a}$ meunier@inln.cnrs.fr 


\section{Introduction}

The transition between the vapour and liquid phases of a material has been for a long time the prototype for first order transitions. Below a critical temperature $T_{c}$, the density, that is the derivative of the pressure $\mathcal{P}$ with respect to the chemical potential $\mu$, is discontinuous at $\mu=\mu_{t}$, where the two phases coexist. The vapour phase is stable below $\mu_{t}$, the liquid phase above. As $T \rightarrow T_{c}$, the transition becomes continuous, the density fluctuations diverge, leading to the well known phenomenon of critical opalescence. Van der Waals theory of condensation predicts these equilibrium properties, and in addition lead to the existence of metastable states, vapour above $\mu_{t}$ and liquid below, experimentally accessible during finite times. In this theory, they correspond to the possibility of analytically continuing the low and high density branches of the isotherm function $\left.\mathcal{P}(\mu)\right|_{T}$ beyond $\mu_{t}$.

All of this can be found in standard textbooks, together with other methods leading to similar descriptions, as well as applications of the same ideas to many systems of physical and/or theoretical interest, which undergo first order transitions. One of the simplest and most extensively studied system is the Ising model. There instead of the pressure, one considers $\left.\mathcal{F}(h)\right|_{T}$, the free energy as a function of the applied magnetic field $h$ at fixed $T$. Below $T_{c}$, the field driven first order transition is located at $h_{t}=0$, and manifested by a discontinuity of the magnetization. As $T \rightarrow T_{c}$, the magnetization vanishes, and its fluctuations measured by the susceptibility, diverge with a critical exponent which is known in 2D. In this particular case however, it has been mathematically proven by Isakov [1] that Van der Waals theory cannot be true: each of the ordered phase free energies possesses an essential singularity at $h=h_{t}$, which makes its analytical continuation ambiguous in the metastability domains where the magnetization remains opposite to the applied field.

In fact, such a singularity in the control parameter at the transition was anticipated a long time ago from the droplet theory of condensation in the version developped by Fisher [2], who attributes to Mayer [3] the first suggestion in this direction. Shortly after, Langer [4] applied the droplet model to the Ising case, emphasizing that the expected singularity was so weak that it could not be experimentally detected. The reader will find in [2, 柾 many references to earlier and related work, and inspiring discussions on the physics behind. Since then, the case has been reexamined many times, with particular concerns about the definition and properties of the so-called droplets, or clusters. Steps in this line of research can be followed 
in, e.g., [0, 6, 07. Its intimate connection with metastability and dynamics at first order transitions has been for a long time a matter of investigations and debate [8, 9], and still is an active field 10, 11, 12, 13, 14, 15].

The basic idea of the approach is that the fluctuations of the system in a stable phase are due to the appearance of droplets of one or several other phases, whose size and number are controlled by the value of the driving parameter. The statistics of these droplets is described as a Mayer's cluster expansion of the thermodynamical function at hand, and the analyticity of the latter follows from the convergence properties of this expansion. A transition occurs at a point of parameter space where the energy/entropy balance favours large clusters of the wrong phase, and the cluster expansion is shown to diverge at such a point, which thus is singular.

In this paper, we investigate similar issues within the bidimensional, $q$ states, Potts model [16] for which we adapt the droplet picture, much in the spirit of [2], using the fact that the transition, here driven by temperature and being first order for $q>4$, becomes continuous as $q \rightarrow q_{c}=4$ where the latent heat and the interfacial tension vanish. The Potts model is interesting per se, as illustrated by the many studies which have been and still are devoted to it, and it seems thus worthwhile to learn more about it. As we shall see, the droplet point of view allows one to put together in a very compact way many of the results accumulated over years, either analytically or by high precision simulations [17, 18] at the transition. It is the case for the relationship between properties of the free energy along the first order transition line $q>4$ and at its critical end point $q_{c}$, a relationship which was evidenced in a series of papers by Bhattacharya et al. 19, 20, 21]. This connection was recently studied in a completely different framework by Cardy 22] who relates the emergence of a critical $q_{c}$ to branching properties of the Potts interfaces between different ordered phases. A better knowledge of the analytic structure of the free energy close to a first order point may also help in other domains of current interest, such as the dynamics, as already mentioned, and the role of disorder for the phase diagram of statistical systems.

Duality relates the disordered phase, stable at high temperature, to the $q$ ordered phases, stable at low temperature. We will mainly focuss on the disordered phase. The paper is organized as follows. In Section (2), we adapt the droplet formulation to the Potts case, and constrain its parameters by requiring consistency, for $q$ values above 4, with the known critical properties at $q_{c}$. In Section (3), we first show that, in a pure phase, the order $n$ energy cumulants $f_{n}$ of the model compare successfully with those measured in [17] 
for $n \leq 8$ and $q=10,15,20$. Only one, $q$-independent, parameter is needed to reproduce all ratios $f_{n} / f_{2}$ given $f_{2}$ at each $q$. This scaling property allows one to construct a universal (i.e. $q$-independent) function $\phi(z)$, which represents the pure phase free energy suitably rescaled, $z$ measuring the distance to the transition point in units of the inverse temperature $\beta$, also rescaled. The function $\phi$ has a simple integral representation from which we derive its analytic properties. It has an essential branch point singularity at $z=0$. We then turn to applications. The associated internal energy distribution for a finite square lattice is studied in Section (4). In particular, rescaling the internal energy and the lattice size leads to a universal distribution from which any $q$-dependence has disappeared. We emphasize consequences for the distribution of the singular structure of the free energy at the transition. Specific finite size effects are predicted, although by assumption the thermodynamical limit of the free energy density has been taken, and compared with those actually observed in a numerical simulation performed at $q=9$. In Section (5), we define the disordered distribution below the transition temperature by reweighting that defined above. From its finite size properties, we determine a size dependent spinodal value $\beta^{*}$ such that the system is metastable in the interval $\beta^{*}>\beta>\beta_{t}$. Inside this interval, which shrinks to zero in the thermodynamical limit, we define a free energy for metastable states. A summary and conclusions are proposed in a last section.

\section{The Potts model and its droplet description}

We first recall a few basic definitions and properties relevant for our discussion of thermodynamical quantities associated with the ferromagnetic $q$-states Potts model. We next summarize the standard droplet description of condensation, and finally adapt it so as to incorporate the known properties of the model at $q \geq 4$.

\subsection{The Potts Model}

The model is defined [16] through the Hamiltonian

$$
H=-J \sum_{<i j>} \delta_{\sigma_{i} \sigma_{j}}
$$

where $\langle i j\rangle$ denotes the pairs of nearest neighbours on a square lattice of area $A=L^{2}$ and $\sigma_{i}$ one of the $q$ possible values of the spin variable at site 
$i$. In the rest of the paper, the energy per link $J$ is set equal to 1 .

The partition function of the system is

$$
\begin{aligned}
Z_{A}(\beta) & =\sum_{\{\sigma\}} \exp (-\beta H(\sigma)), \\
& =\sum_{E} \Omega_{A}(E) \exp (-\beta A E)
\end{aligned}
$$

where $\beta$ is the inverse temperature, $E \in[-2,0]$ the energy density per site, and $\Omega_{A}$ the corresponding number of states. It is equivalent to say that the energy probability density is

$$
P_{\beta, A}(E)=\frac{1}{Z_{A}(\beta)} \Omega_{A}(E) \exp (-\beta A E) .
$$

For convenience, we shall call "free energy" the quantity

$$
F_{A}=\frac{1}{A} \log \left(Z_{A}\right),
$$

while strictly speaking $F_{A}$ is $-1 / \beta$ times the standard free energy density. Its thermodynamical limit $F$ is its limit as $A \rightarrow \infty$. The finite size microcanonical entropy density $S_{A}(E)$ and its thermodynamical limit $S(E)$ are defined by

$$
\begin{aligned}
S_{A}(E) & \equiv \frac{1}{A} \log \left(\Omega_{A}\right), \\
& =F_{A}(\beta)+\frac{1}{A} \log \left(P_{\beta, A}(E)\right)+\beta E, \\
S(E) & =\lim S_{A}(E), \quad A \rightarrow \infty .
\end{aligned}
$$

A numerical simulation provides the energy distribution $\Omega_{A} e^{-\beta A E}$ up to a numerical factor, and thus the entropy $S_{A}$ up to an additive constant.

A review of many of the known properties of the Potts model can be found in [23]. A transition occurs at an inverse temperature $\beta_{t}$ given by [24]

$$
\beta_{t}=\log (\sqrt{q}+1) \text {. }
$$

It is first order for $q>4$, and second order at and below the end point $q_{c}=4$, where it is characterized, e.g., by the critical indices $\alpha$ and $\nu$ for the specific heat and the correlation length respectively . For future reference, we recall that

$$
\begin{aligned}
& \alpha=2 / 3, \\
& \nu=2 / 3 .
\end{aligned}
$$


In the thermodynamical limit, the system is either in the disordered phase, for $\beta<\beta_{t}$, or in one of the $q$ degenerate ordered phases for $\beta>\beta_{t}$. When necessary, a quantity referring to an ordered or to the disordered phase will receive a superscript $o$ or $d$ respectively. So $F^{(o)}\left(\right.$ resp. $\left.F^{(d)}\right)$ denotes the ordered (resp. disordered) free energy, a well defined function of $\beta$ for $\beta>\beta_{t}$ (resp. $<\beta_{t}$ ). The transition point is the $\beta$ value where the two free energies are equal: $F^{(o)}\left(\beta_{t}\right)=F^{(d)}\left(\beta_{t}\right)$. For $q>4$ where the transition is first order, one expects on very general grounds that at $\beta_{t}, F^{(o)}$ (resp. $\left.F^{(d)}\right)$ has finite left (resp. right) derivatives to any order $n$ with respect to $\beta$. They give $(-1)^{n}$ times the cumulants $f_{n}^{o, d}$ of the internal energy associated with each of the pure phases $o$ or $d$. We shall be interested in the analytic continuation of the free energies in the complex $\beta$ plane at $q>4$ fixed, which will consist in giving a meaning to the formal expansion:

$$
F^{(o, d)}(\beta)=\sum_{n=0}^{\infty}(-1)^{n} f_{n}^{o, d}\left(\beta-\beta_{t}\right)^{n} / n ! \quad .
$$

The two functions $F$ are related to each other by duality [23]. If

$$
(\exp (\tilde{\beta})-1)(\exp (\beta)-1)=q,
$$

then

$$
F^{(o)}(\tilde{\beta})=F^{(o)}(\beta)-2 \log ((\exp (\beta)-1) / \sqrt{q}) .
$$

We choose to study $F^{(d)}$ for concretness, and consider the $d \rightarrow o$ transition as a condensation process where droplets of aligned spins inside a disordered bulk tend to grow as $\beta$ approaches $\beta_{t}$ from below. The droplet model consists in describing the system at equilibrium as a statistical distribution of non interacting droplets.

\subsection{The Droplet Picture}

We closely follow Fisher [2]. The transposition from vapour condensation to the $d \rightarrow o$ Potts transition at $q>q_{c}$ produces the disordered phase free energy $F^{(d)}(\beta)$ as a function of the inverse temperature. A droplet of size $\ell$ (its area) is a connected domain of $\ell$ sites with the same spin value. The model assumes that it has an effective perimeter scaling as $\ell^{\sigma}$, where the exponent $\sigma$, smaller than 1 (no fully ramified clusters), but possibly larger than the geometrical value $1 / 2$ [25], accounts for the appearance at fixed $\ell$ of many different shapes and topologies. This is controversial and calls for comments to be made later. The free energy can then be written: 


$$
\begin{aligned}
F^{(d)}(\beta) & =c \sum_{\ell=1}^{\infty} \ell^{-\tau} x^{\ell^{\sigma}} y^{\ell} \\
y & =\exp \left(\beta-\beta_{t}\right) \\
x & =\exp (-\omega)
\end{aligned}
$$

with

$$
\omega>0, \quad 1 / 2<\sigma<1, \quad \tau>0 .
$$

The parameter $c$ fixes the normalisation and $\ell^{\tau}$ is a correction to the area and perimeter dependences [4, 26]. A priori, the parameters $\omega, \sigma, \tau$ and $c$ are functions of $q$, a question which we examine in the next subsection. Eqs. (14 16) are interpreted as follows. The free energy is the sum over $\ell$ of contributions coming from all clusters of size $\ell$. The quantity $y$ plays the role of the activity in gas condensation or of $\exp (-h)$ in the Ising problem, and $\omega$ denote an energy density per unit of effective perimeter (effective interface tension between the interior and the exterior of a droplet).

Each term in the above sum is proportional to the probability of an ordered cluster of size $\ell$ in the system. Due to inequalities (17), the factor $y^{\ell}$ implies that for $\beta<\beta_{t}$ the probability of arbitrary large clusters is exponentially small. The disordered phase is stable there, the series and all its derivatives with respect to $\beta$, converge. The converse is true for $\beta>\beta_{t}$. It follows that the disordered free energy $F^{(d)}$ is analytic in the half-plane $\operatorname{Re}(\beta)<\beta_{t}$ and has an essential singularity at $\beta_{t}$.

As a side remark, we note that in its Kasteleyn-Fortuin formulation [27], the Potts model is a model of satisfied or unsatisfied links. The temperature dependence of the partition function is carried by weigths $\bar{y}^{l}$, with $\bar{y}=(\exp (\beta)-1) / \sqrt{q}, \ell$ being the number of satisfied links forming a connected cluster. The droplet picture applied to the link representation leads to equations similar to (14,15) with $y$ replaced by $\bar{y}$. Close to the transition, $y \simeq \bar{y} \simeq 1$ up to order $\left(\beta-\beta_{t}\right)$ terms, so that using $y$ or $\bar{y}$ makes no difference for the leading singularity: It has the same structure in $b=\log \bar{y}$ and in $\beta-\beta_{t}$.

Following Fisher [2], we now consider the system close to the critical point $q_{c}$, and fix the parameters $\omega, \sigma$ and $\tau$. 


\subsection{Fixing the Parameters from the Critical Point}

We go on with the disordered phase free energy, and from now on omit the $d$ superscript. The energy cumulant of order $n$ is given by

$$
f_{n}=\left.(-1)^{n} \frac{d^{n}}{d \beta^{n}} F\right|_{y=1}
$$

with F given by Eq.(14). At least for large enough orders, the series obtained for $f_{n}$ can be given a closed form, by replacing the discrete sum over $\ell$ by an integral [2, 此. The result is

$$
\begin{aligned}
f_{n} & =c(-1)^{n} \int_{0}^{\infty} d \ell \exp \left(-\omega \ell^{\sigma}\right) \ell^{n-\tau} \\
& =\frac{c(-1)^{n}}{\sigma \omega^{(n-\tau+1) / \sigma}} \Gamma((n-\tau+1) / \sigma) .
\end{aligned}
$$

Hence all the derivatives of $F$ exist at the transition, but due to $\sigma<1$ the convergence radius of its Taylor series (11) in $\left(\beta-\beta_{t}\right)$ is zero, a characteristic situation for an essential singularity at $\beta_{t}$. It also follows from (20) that the scale in $\beta-\beta_{t}$ relevant for the behaviour of $\mathrm{F}$ close to the transition is

$$
\beta_{0}=\omega^{1 / \sigma} .
$$

On another hand, from an expansion to order 10 in $1 / \sqrt{q}$ of the free energy, it was empirically found in [19, 20, 21], and confirmed by expansions recently pushed to order 23 [28], that at least in a "low" $q$ domain, in fact extending up to $q \approx 30$ or more, one has approximately

$$
\begin{aligned}
f_{2} & \propto \xi \\
f_{3} / f_{2} & \propto \xi^{3 / 2},
\end{aligned}
$$

where $\xi$, the correlation length of the Potts model, is exactly known [29, 30] and grows extremely rapidly as $q$ decreases towards $q_{c}$. In 19, 20, a tentative explanation for such behaviours was that $a t q_{c}, \xi$ and the most singular part $F_{\text {sing. }}$ of $F$ are respectively proportional to $\left|\beta-\beta_{t}\right|^{-\nu}$ and $\left|\beta-\beta_{t}\right|^{2-\alpha}$, so that Eqs. 22, 23) hold there, due to (10), and thus perhaps in some neighborhood of the critical point. Together with $(20,21)$, this invites us to postulate

$$
\begin{aligned}
\omega & =f / f_{2} \propto 1 / \xi \\
\sigma & =2 / 3, \\
\tau & =7 / 3,
\end{aligned}
$$


with $f$ approximately constant with respect to $q$.

Note that if $\omega$ in (14,16) is interpreted as an effective interfacial tension between the interior and the exterior of a droplet, its postulated behaviour (24) fits nicely that of the true $o-d$ interface tension $\Sigma$, known to be equal to $1 / 2 \xi$ [30], and also determined numerically via a study of phase coexistence at $\beta_{t}$ [18]. We remark that at $q=q_{c}$ the same behaviour $\Sigma \xi \rightarrow$ cst. as $\beta \rightarrow \beta_{c}$ follows from the scaling relation $\mu=\nu, \mu$ being the Widom exponent for the interface tension [31]. Why it also holds for $\omega \xi$ remains however unclear since whereas $\Sigma$ is an energy density per unit length along a straight boundary, $\omega$ is here related to an effective boundary whose size scales as a length to the power $2 \sigma=4 / 3(25)$.

As discussed in details by Binder [5] for the Ising case, taking $\sigma \neq 1 / 2$ is quite questionable, and in fact incorrect if one insists that the droplets of the model are the standard geometric clusters which can be drawn on a lattice. This is confirmed by the exact results of [6, 7] about the probability of such clusters, and of Isakov [1] on the cumulants of the Ising model at sufficiently low temperature. Because their derivation is essentially of geometrical nature, we see no reason why they should not apply to the Potts case. A physical interpretation of $\sigma>1 / 2$ has been proposed by Binder. For the time being, we take $\sigma=2 / 3$ as an effective parameter describing some neihborhood of the critical end point, and show that anyway it accounts for many observed facts. This point will be discussed again in our conclusions (Section (6)).

\section{The Cumulants confronted to Numerical Data. The Free Energy}

The arguments borrowed from [19, 20] to guess $f_{3} / f_{2}$ Eq.(23) were furthermore applied in these references to cumulants of any order by successive derivations of $F_{\text {sing. }} \propto\left(\beta_{t}-\beta\right)^{2-\alpha}$ and the subsequent replacement of $\left(\beta_{t}-\beta\right)$ by a constant times $\xi^{-1 / \nu}$. As a result, the series (11) converged, providing an ansatz for $F^{(d)}$ [21] analytic up to a value $\beta^{*}>\beta_{t}$, which could be interpreted as the end of a metastability region (spinode), in clear contradiction with any droplet approach. 


\subsection{The Potts model cumulants}

Let us compare the model predictions for the energy cumulants with the results of the high accuracy simulation of Janke and Kappler 17, who give the pure phase $f_{n}$ up to order 8 at $\beta_{t}$. We are concerned with $n \geq 2$ only, i.e. with those cumulants which diverge at the critical point. Anyway, $f_{0}$ and $f_{1}$ are exactly known [24], and irrelevant for the study of a single phase. Since the normalisation constant $c$ remains undetermined and since $f_{2}$ is supposed to carry most of the $q$-dependence of $\omega(24)$, we rewrite (20) as

$$
\frac{f_{n}}{f_{2}}=(-1)^{n}\left(\frac{f_{2}}{f}\right)^{(n-2) / \sigma} \frac{\Gamma((n-\tau+1) / \sigma)}{\Gamma((3-\tau) / \sigma)}, \quad n \geq 2,
$$

that is, if the conjectures $(25,26)$ are used

$$
\begin{aligned}
& \frac{f_{n}}{f_{2}}=(-1)^{n} S_{n} \Gamma(3 n / 2-2), \\
& S_{n}=\left(\frac{f_{2}}{f}\right)^{(3 n / 2-3)} .
\end{aligned}
$$

This is the basic equation of our paper. It determines all the $f_{n}$ 's, $n>2$ as a function of $f_{2}$ at the price of one free parameter $f$ only. We fitted Eq. (28) to the data [17], fixing $f_{2}$ at the central value measured for each $q=10,15$ and 20, $n$ varying from 3 to 8 . We obtained a $\chi^{2} /$ d.o.f. equal to 1.3 for 17 degrees of freedom with

$$
f=0.295 \pm 0.003
$$

The excellent agreement is illustrated in Fig.[1] where the numerical data [17] for $(-1)^{n} f_{n} /\left(f_{2} S_{n}\right), n \leq 8$ are compared to $\Gamma(3 n / 2-2)$ (continuous line) and plotted against the order $n$. Also indicated are the orders of magnitude estimated for $n=9$ and 10. In order to appreciate whether the values $(25,26)$ of $\sigma$ and $\tau$ are actually requested by the numerical data, we

have repeated the fit letting also these parameters free. The new fit requires

$$
\begin{aligned}
\sigma & =0.67 \pm 0.01 \\
\tau & =2.34 \pm 0.10 \\
f & =0.28 \pm 0.02
\end{aligned}
$$

with $\chi^{2} /$ d.o.f. $=1$ for 15 d.o.f.., in perfect agreement with our conjecture. 
This nicely confirms the existence of a large domain of "low" $q$ values where the fluctuations of the system are strongly influenced by the critical properties at $q_{c}$. The Fisher droplet model, supplemented by our conjecture that the whole $q$-dependence is embedded in $f_{2}$, gives them an economical and accurate description. We note that the ansatz [21] predicts much smaller values for the highest order cumulants, missing $f_{8}$ by a factor about thirty. These results not only support the existence of an essential singularity in the free energy, but also show that this singularity does lead to detectable effects. We now proceed to construct the free energy explicitly .

\subsection{The Disordered Phase Free Energy}

The free energy can be reconstructed either directly from the droplet formulation (14), replacing the sum by an integral as done in [4], or from the asymptotic series (11) with $f_{n}$ given by (27) or (28) and the $\Gamma$-function replaced by its integral representation (Borel resummation). In view of the results of the previous subsection, it is natural to introduce the following rescaled free energy $\phi$, expressed as a function of a rescaled temperature $z$,

$$
\begin{aligned}
\phi(z) & \equiv\left(\frac{f_{2}}{f}\right)^{2 / \sigma} \frac{1}{f_{2}}\left(F(\beta)-F\left(\beta_{t}\right)-f_{1}\left(\beta-\beta_{t}\right)\right), \\
z & \equiv-\left(\beta-\beta_{t}\right)\left(f_{2} / f\right)^{1 / \sigma} .
\end{aligned}
$$

With this choice of normalizations, we have $\phi(0)=\phi^{\prime}(0)=0$ and $\phi^{\prime \prime}(0)=1$. For generic values of $\sigma$ and $\tau, \phi$ reads

$$
\phi(z)=\frac{1}{\Gamma((3-\tau) / \sigma)} \int_{0}^{\infty} \frac{d t}{t^{(\sigma+\tau-1) / \sigma}} e^{-t}\left(e^{-z t^{1 / \sigma}}-1+z t^{1 / \sigma}\right),
$$

and specializing to the values $(25,26)$ of $\sigma$ and $\tau$, we finally get

$$
\phi(z)=\int_{0}^{\infty} \frac{d t}{t^{3}} e^{-t}\left(e^{-z t^{3 / 2}}-1+z t^{3 / 2}\right) .
$$

This function is obviously holomorphic for $\operatorname{Re} z>0$, which includes the stability region $\beta<\beta_{t}$ of the disordered phase. It can be analytically continued in the complex $z$-plane by deforming the integration contour in t. The only singularity is a branch point at $z=0$, easy to characterize. The contribution to $\phi$ from any finite $t$ interval $\left[0, t_{0}\right]$ is an entire function, as well as that from $t_{0}$ to $\infty$ of the linear part (in $z$ ) of the integrand. Hence the singular part of $\phi$ is that of

$$
\phi(z)=\int_{t_{0}}^{\infty} \frac{d t}{t^{3}} e^{-t-z t^{3 / 2}}
$$


for any positive $t_{0}$. Next, as $z$ is continued to $z=|z| \exp ( \pm i \pi)$, one may continuously move the contour $\left[t_{0},+\infty\right]$ to $\left[t_{0}, \mp i \infty\right]$ leading to a discontinuity along the negative real axis

$$
\begin{aligned}
\Delta & \equiv \phi(-|z|+i \epsilon)-\phi(-|z|-i \epsilon), \\
& =-\int_{t_{0}-i \infty}^{t_{0}+i \infty} \frac{d t}{t^{3}} e^{-t-z t^{3 / 2}} .
\end{aligned}
$$

In the following, we define $\phi(z)$ by (37) in the whole complex plane cut along the negative real axis, on which $\phi$ acquires an imaginary part. For $|z|$ small, the above integral can be estimated by steepest descent, giving

$$
\operatorname{Im} \phi(-|z|+i \epsilon)=-\sqrt{\pi}\left(\frac{3|z|}{2}\right)^{5} \exp \left(-\frac{4}{27 z^{2}}\right) I(z),
$$

where the function $I(z)$, easy to evaluate numerically, goes smoothly to one as $z$ goes to 0 along the negative real axis. All this is quite similar to the analysis performed by Langer [四] for the Ising case, where the magnetic field plays the role of $z$. Whenever a precise evaluation of $\phi(z)$ is needed, one can either numerically integrate the analytic continuation of (37) using suitable contours, or use its large $|z|$ expansion, which is found to be:

$$
\begin{aligned}
\phi(z) & =\gamma / 6-3 / 4-\log (z) / 3-2 z \sqrt{\pi}+S \\
S & =\frac{2}{3} z^{4 / 3} \sum_{q \geq 0, \neq 2}^{\infty} \frac{(-1)^{q}}{q !} z^{-2 q / 3} \Gamma\left(\frac{2 q-4}{3}\right),
\end{aligned}
$$

where $\gamma$ is the Euler's constant. The derivation of this expansion can be obtained by a Sommerfeld-Watson method. One starts from the formal series expansion of $\phi$ in $z^{n}$, truncated at some large $n$ value $N$. The sum $\sum_{0}^{N}(-1)^{n} a_{n}$ is replaced by the integral $1 /(2 i) \oint d \nu a_{\nu} / \sin (\pi \nu)$ over a contour encircling all integers from 0 to $N$. After sending $N$ to $\infty$ the contour is deformed so as to encircle the poles of the integrand situated on the negative real axis, and the residue formula is finally applied. A similar expansion for the second derivative of $\phi$ with respect to $z$ is much easier to derive starting from

$$
\begin{aligned}
\phi^{\prime \prime}(z) & =\int_{0}^{\infty} d t e^{-t-z t^{3 / 2}} \\
& \equiv z^{-2 / 3} \int_{0}^{\infty} d t \exp \left(-t / z^{2 / 3}-t^{3 / 2}\right)
\end{aligned}
$$


and expanding the latter integral in powers of $z^{-2 / 3}$ which yields a convergent series in this variable [32].

As we have seen, the closed form (37) of the thermodynamical free energy incorporates the information acquired upon the $\sim 10$ first energy cumulants in simulations performed on large but finite lattices during finite (MonteCarlo) times. That it makes sense is justified by exact results [34, which, in short, state that on a finite lattice, $1 / A \log \left(Z_{A}^{(d)}\right)$ gives the thermodynamical limit $F^{(d)}$ up to corrections which are exponentially small in the linear size of the lattice. In contrast, the next section will illustrate that the energy distribution $P_{A}(E)$ on a finite lattice exhibits strong finite size effects, specific to the singularity structure of the thermodynamical free energy, and corroborated by a numerical simulation at $q=9$. The procedure followed to study $P_{A}$ is similar to that used in [21].

\section{Finite Size Effects and Scaling Properties in the Internal Energy Distribution}

\subsection{The Probability Density for the Energy}

Given the rescaled free energy $\phi(z)$, the probability density for observing a lattice averaged energy density $\mathrm{E}$ in a supposedly disordered bulk phase at $\beta=\beta_{t}$, on a lattice of area $A$, is obtained by inverse Laplace transform using Eqs. (3, 4, 5). We find

$$
P_{\beta_{t}, A}(\epsilon)=\frac{A_{r}}{2 i \pi} \int_{\bar{z}-i \infty}^{\bar{z}+i \infty} d z \exp \left(A_{r}(\phi(z)-\epsilon z)\right),
$$

where the rescaled energy, area and inverse temperature $\epsilon, A_{r}$ and $z$ respectively are defined as

$$
\begin{aligned}
\epsilon & =\frac{1}{f}\left(\frac{f_{2}}{f}\right)^{1 / 2}\left(E-E^{(d)}\right), \\
A_{r} & =f\left(\frac{f}{f_{2}}\right)^{2} A, \\
z & =-\left(\frac{f_{2}}{f}\right)^{3 / 2}\left(\beta-\beta_{t}\right) .
\end{aligned}
$$

The symbol $E^{(d)}$ (or $E^{(o)}$ ) represents the exactly known internal energy of the disordered (or ordered) phase in the thermodynamical limit [23]. In 
the rest of this paper, and depending on the context, we will use either the physical $E, A$ and $\beta$ variables, or their rescaled forms $\epsilon, A_{r}$ and $z$. For economy of notations however, we will often keep the same name for functions of them. The variable $\mathrm{E}$ (or $\epsilon$ ) is considered as continuous, $\beta$ (or $z$ ) as unlimited in the imaginary direction. Eq. (47) maps the physical region $E \in[-2,0]$ over a large interval in $\epsilon$, the more so $f_{2}$ is large (see Eq. (47), that is $q$ close to 4 . Since on large lattices $P$ is very sharply peaked around $\epsilon=0$, it will be justified (and convenient) to consider also $\epsilon$ as unlimited, unless specified. Due to the analyticity properties of $\phi(z)$, the integral (46) does not depend on $\bar{z} \geq 0$.

With the above prescriptions, one can verify that $P_{\beta_{t}, A}(\epsilon)$ is a probability density, normalized to one with respect to integration over $\epsilon$, that $<\epsilon>=0,<\epsilon^{2}>=1$, and more generally that the cumulants of $\epsilon$ for the density $P_{\beta_{t}, A}$ have exactly the values assigned by Eqs.(34, 35, 28), a very powerful check for numerical integration of (46). Any explicit $q$-dependence has disappeared, and the effective area $A_{r}$ is the only external parameter. Its relation (48) to the physical area $A$ predicts the scaling property that different $q$-values lead to the same $P_{\beta_{t}, A}(\epsilon)$ if they are measured on lattices of linear size proportional to $f_{2}$ (i.e. approximately to $\xi$ according to (22), which sounds reasonable). We note that the largest $L$ values used in [17 for $q=[10,15,20]$ were precisely chosen roughly proportional to $\xi$.

This scaling behaviour is independent of the particular form of $\phi(z)$, and we turn to the study of those properties of the distribution which are specific to our construction. The results of this section are all summarized through their application to the case $q=9$ with $L=80,120$ and 200, shown in Fig. [2]. The rescaling of $A=L^{2}$ to $A_{r}$ (48) requires the knowledge of $f_{2}$ at $q=9$. There the correlation length is 14.9 [30], and we evaluate $f_{2}$ from its value at $q=10$ [17] by $(22)$, finding $f_{2}(q=9)=12.8$. The quantity shown in Fig.[2] is

$$
q_{\beta_{t}, A}(\epsilon)=\frac{1}{A_{r}} \log \left(\frac{P_{\beta_{t}, A}(\epsilon)}{\sqrt{A_{r}}}\right)
$$

The continuous lines correspond to the numerical integration of (46), and the other ones to various analytical approximations described below. 


\subsection{The Distribution above the Disordered Peak, $\epsilon>0$.}

For $\epsilon \geq 0$, the saddle point equation for the integral (46), namely

$$
\frac{d \phi}{d z}=\epsilon
$$

has a unique, non negative solution $z_{s}(\epsilon)$. Taking $\bar{z}=z_{s}$ in the definition (46) makes the numerical integration easy. The saddle point estimate of $P_{\beta_{t}, A}$, valid at large $A_{r}$, is

$$
P_{\beta_{t}, A}^{(s)}=\sqrt{\frac{A_{r} \phi^{\prime \prime}(z)}{2 \pi}} \exp \left(A_{r}\left[\phi(z)-z \frac{d \phi}{d z}\right]\right), \quad z=z_{s}(\epsilon), \quad \epsilon>0 .
$$

In this approximation, the thermodynamical limit of $q_{\beta_{t}, A}$ (50) exists for any non negative $\epsilon$, and defines, up to a linear term in $\epsilon$, a non trivial, concave, microcanonical entropy density $S(E)$. Moreover, for $\epsilon$ large, an estimate $z_{a s}$ of $z_{s}$ can be obtained, by solving Eq.(51) analytically when the two leading terms only of the large $|z|$ expansion (42) are kept. Then $\phi\left(z_{a s}\right)$ and $\phi^{\prime \prime}\left(z_{a s}\right)$ are computed at the same order, and plugged into (52), avoiding any integration. The corresponding algebraic expressions are quite lengthy, and not here. They are used for Fig.[2], showing that the approximation is very good in practice shortly after $\epsilon=1$. Farther away, these expressions show that $q_{\beta_{t}, \infty}(\epsilon)$ become proportional to $-(\epsilon+2 \sqrt{\pi})^{4}$. This means an extremely fast decrease of $P(\epsilon)$ on the right hand side of the peak, much faster than any Gaussian.

\subsection{The Distribution below the Disordered Peak. Scaling at $\epsilon<0$}

The side $\epsilon<0$ is more unusual, its properties reflecting the tendancy of the disordered system to order. As $\epsilon$ passes through 0, the solution of (51) disappears from the first Rieman sheet, and we need to optimize the integration contour in (46) differently. Given $\epsilon$, we move it to a contour $z \equiv x \pm i y(x, \epsilon)$ passing through 0 and determined by the condition

$$
\operatorname{Im}(\phi-\epsilon z)=0 .
$$

In practice, because $\operatorname{Im}(\phi(x))$ is never large before the integrand in (46) gets exponentially damped at large $|x|$, we expand $\phi(x+i y)$ in $y$ to second order (note that $\phi$ is analytic and thus expandable at any non zero $\mathrm{x}$ ), and solve 
(53) for $y$ as a function of $\phi(x)$ and of its two first derivatives along the real axis. Along this approximate path, the integrand does not oscillate too much, allowing smooth numerical integration, whose result is drawn as continuous lines in Fig. [2].

We extended the curves down to unrealistically small probabilities, in order to illustrate the peculiarities of the distribution, both in shape and in size dependence. Compared to the right hand side of the peak, the curve on the left is much broader and it broadens substantially as $L$ increases. A similar behaviour of the Ising magnetization distribution at zero field has been analyzed in 10]. We now correlate these features to the singular part of $\phi(z)$, via an analytical estimate of $P_{\beta_{t}, A}(\epsilon)$ valid at $\epsilon<0$.

The integral (46) is also equal to that of minus twice the imaginary part of the same integrand above the cut:

$$
P_{\beta_{t}, A}(\epsilon)=-\frac{A_{r}}{\pi} \int_{-\infty}^{0} d x \exp \left(A_{r}(\operatorname{Re} \phi(x)-\epsilon x)\right) \sin \left(A_{r} \operatorname{Im} \phi(x)\right) .
$$

For $-\epsilon$ not too small, the integrand is exponentially damped when $|x|$ increases, the more so $A_{r}$ is large, while it vanishes at small $|x|$ due to the behaviour (41) of $\operatorname{Im} \phi$. Thus for $A_{r}$ large, but fixed, and $|x|$ small enough, $\operatorname{Im} \phi$ can be replaced by its approximation (41) and $\sin \left(A_{r} \operatorname{Im} \phi\right)$ by its argument. The existence of a saddle point $x_{s}$ follows. Neglecting $R e \phi\left(x_{s}\right)$ in front of $\epsilon x_{s}$, one finds

$$
\begin{aligned}
x_{s} & =-\left(\frac{8}{27 A_{r}|\epsilon|}\right)^{1 / 3}, \\
\frac{1}{A_{r}^{2}} P_{\beta_{t}, A} & =\frac{2}{3}\left(A_{r}|\epsilon|\right)^{-7 / 3} \exp \left(-\left(A_{r}|\epsilon|\right)^{2 / 3}\right), \quad \epsilon<0 .
\end{aligned}
$$

Hence we have the remarkable scaling prediction that, up to a factor $A^{2}$, the distribution depends on both the energy and the size through the single variable $A_{r}|\epsilon|$. Moreover, the argument of the exponential damping of the probability is not extensive and its $\epsilon$-dependence is less than linear. These two properties explain the observed broadening of the distribution, and their very derivation emphasizes the role of a non vanishing imaginary part. Fig.[2] shows that the approximation (56) works quantitatively, the more so the product $\left|A_{r} \epsilon\right|$ is large, which actually insures that $x_{s}$ and thus $A_{r} \operatorname{Im} \phi$ are small. The behaviour (56) will be important for our discussion of metastability (see Section (5)). 


\subsection{A Numerical Simulation at $\mathrm{q}=9$}

We have just seen that the scaling law (56) sets in for $A_{r}|\epsilon|$ sufficiently large. Hence a numerical simulation designed to check it should accumulate reasonable statistics for events whose probability relative to the peak value is quite small. This means very extensive simulations, typically of the size of those of Refs. 17, 18]. However, Fig.[2] shows that substantial finite size effects already appear in $q_{\beta_{t}, A}(\epsilon)$, Eq.(50), in a crossover region in between $\epsilon=0$ and the above mentioned scaling domain. In order to complement the comparison with simulations made for the cumulants (Section (3.1)), by a direct study of finite size effects in energy distributions, we performed a medium size simulation for $q=9$, where the correlation length is about 1.4 larger than at $q=10$, and on lattices of sizes $L=80,120$, and 200 .

We used a Glauber type of algorithm. The site to be updated is chosen at random and its new spin value determined according to its Boltzmann weight. At $\beta=\beta_{t}=\log (4)$ we performed runs consisting for each $L$ of about $10^{6}$ sets of $L^{2}$ random hits at the lattice sites. We measured the internal energy density $E$ every 10 sets, thus collecting a sample of $\simeq 10^{5}$ configuration energies for each $\mathrm{L}$ value. Starting from a disordered configuration (spin chosen at random on each site), the histogram obtained is proportional to the normalized probability density $P_{\beta_{t}, A}$ discussed above. For comparing the numerical data to the model distribution, Fig.(3), we divide each of them by its value at a reference energy which we choose to be $E_{0}=-1$, and as a function of the internal energy $E$ we plot the quantity

$$
p_{\beta_{t}, A}(E)=10^{3} \frac{1}{A} \log \left(\frac{P_{\beta_{t}, A}(E)}{P_{\beta_{t}, A}\left(E_{0}\right)}\right) .
$$

Here we restored the physical variables using Eqs.(47, 48) with $f_{2}=12.8$ as explained in Section (4.3).

At each value of $E$, the error quoted is estimated from the fluctuations around the mean observed in subsamples of $10^{3}$ to $10^{4}$ measurements. In this range, the error was found to depend weakly upon the bin size, in agreement with an estimated autocorrelation length of order $10^{2}$ for the average energy (i.e. in the peak region; it increases away of it). For the clarity of the figure, we suppressed the data points for which the statistics is too small to bring any useful information.

We see on Fig.[3] that both the shape in energy and the dependence in size observed in the simulation are correctly reproduced by our construction, 
an absolute prediction since the only parameter $f$ was previously fixed from the study in section (3.1) of the cumulants at $q \geq 10$. There may be a tendancy for the size dependence to be too strong in the model. We do not consider it as significant enough to justify a retuning of $f$ or $f_{2}$, the more so we did not include in the errors shown that due to the fluctuations at the reference energy $E_{0}$. This error induces an overall uncertainty in the relative vertical positions of the curves. We cannot exclude either that finite size effects, in the sense of a residual $L$ dependence in the free energy, are still present at the lowest $L$.

We conclude that the adequacy of the model to predict quantitatively the strong size dependence observed in the disordered phase energy distribution below the peak, as well as its (nearly) size independent shape above the peak, constitutes a good evidence in favour of the analytical structure of the free energy inherent to the droplet picture.

\section{Beyond the transition temperature. Metasta- bility}

Metastability of the disordered phase refers to the possibility for the system to stay disordered above $\beta_{t}$. Standard phenomenology à la Van der Waals, or Landau mean field theory, would state that the thermodynamical average energy density, which below $\beta_{t}$ coincides with $\langle E\rangle^{d}(\beta)=$ $-d / d \beta F^{(d)}(\beta)$, can be continued up to a so-called spinodal value $\beta_{s p}$, separating a metastable from an unstable region.

In the droplet picture, as well as in field theoretic approaches, the point $\beta_{t}$ is a branch point, and analytic continuation above $\beta_{t}$ is ill-defined. An important question, not considered in this paper, concerns the associated dynamics. An extensive review can be found in [8]; see also [11]. This field is still subject to active research in the context of Ising-like or liquid/vapour transitions. After Langer [ [ 1 , 35] and followers, the nucleation rate of a metastable state is proportional to the imaginary part of the free energy along the cut. Most of the recent results concern the Ising case (See for example [36, 37, 38]). To our knowledge, these dynamical aspects have not been studied for the Potts model above $q_{c}$. Here we limit ourselves to a discussion of which thermodynamical properties can be assigned the metastable states, starting from these features of energy distributions which we found in Section (4) as specifically due to the singular structure of the free energy. For the Ising case, related considerations can be found in [10]. 


\subsection{Energy Distribution away from $\beta_{t}$ by Reweighting}

On a finite lattice, the extrapolation of a given energy distribution from $\beta_{t}$ to any (larger or smaller) $\beta$ is unambiguously defined by the corresponding reweighting of the Boltzmann factor. Hence, if $P_{\beta_{t}, A}(E)$ is the pure disordered phase distribution, then the (unnormalized) distribution at $\beta$ is (we write it both in physical and rescaled variables (47, 48, 49)

$$
\begin{aligned}
D_{\beta, A}(E) & =\exp \left(-A\left(E-E^{(d)}\right)\left(\beta-\beta_{t}\right)\right) P_{\beta_{t}, A}(E) . \\
D_{\beta, A}(\epsilon) & =\exp \left(A_{r} \epsilon z\right) P_{\beta_{t}, A}(\epsilon) .
\end{aligned}
$$

The reweighting (59) is often used to smoothly interpolate, or slightly extrapolate numerical data taken at some fixed $\beta$ point (here at $\beta_{t}$ ). The results of [17] show that it is feasible to perform a high statistics simulation at $\beta_{t}$ without tunneling from one phase to another, so that an accurate numerical determination of the pure phase $P_{\beta_{t}, A}(E)$ was obtained.

We got this distribution for finite lattices from a theoretical ansatz for the thermodynamical limit of the free energy, and now get its continuation beyond $\beta_{t}$ by (59). The generic shapes of the reweighted distributions as $\beta$ is varied are shown in Fig. [4] for $q=9$ and $L=120$. We comment them in the light of the results of Section (4). For $\epsilon$ positive, $P_{\beta_{t}, A}$ decreases faster than a Gaussian (section 4.2), and the only effect of reweighting there is to displace the peak position, whatever the sign of $z$ is. For $\epsilon$ and $z$ both negative (see sections 4.3 and 4.4), the exponential growth of the Boltzmann factor eventually wins over the smoother behaviour (56) of $P_{\beta_{t}, A}$. Hence, under overcooling the distribution finally blows up for $|z|$ large enough, leading to a minimum at $E=E_{m}$, visible in the upper curve on the left of Fig.[4], which exhibits the cubic like shape typical of a metastable situation. In between $E_{m}$ and the location $E_{M}$ of the maximum, all curves have an inflexion point at the same value $E=E^{*}$ : It is the inflexion point of the (finite size) microcanonical entropy $S_{A}(E)$.

The depth of the minimum relative to the peak height is a measure of the barrier to be crossed by the system in order to flip to its stable state, and thus controls the lifetime of the metastable state. There is no meaning in considering the distribution (59) below $E_{m}$, where for the least the contributions of the ordered states, obtainable from ours by duality, and of mixed phase configurations [18] must be included. As a definition, we take the distribution (59) cut below $E_{m}$ as representative of the metastable disordered phase. 
On a given lattice, the barrier disappears when $\beta$ becomes equal to $\beta^{*}$, the slope at $E^{*}$ of

$$
d_{\beta, A}(E) \equiv \frac{1}{A} \log \left(D_{\beta, A}(E)\right) .
$$

The size dependent point $\left(\beta^{*}, E^{*}\right)_{A}$, which plays the role of the classical spinodal point, is thus determined by:

$$
\begin{aligned}
\frac{\partial^{2}}{\partial E^{2}} d_{\beta, A}(E)_{\left.\right|_{E=E^{*}}} & =0 \\
\frac{\partial}{\partial E} d_{\beta, A}(E)_{\left.\right|_{E=E^{*}}} & =\beta^{*}-\beta .
\end{aligned}
$$

At $L=120$ and $q=9$, again chosen for illustration, we find from the model distribution at $\beta_{t}$ (the dotted curve of Fig.[4])

$$
\begin{aligned}
E^{*} & =-1.104 \\
\beta^{*} & =\beta_{t}+3.4410^{-3}=1.38973 .
\end{aligned}
$$

Our statistics at $\beta_{t}$ (Section (4.4)) are not sufficient to give significant results by reweighting, and we explored the above issue by new simulations in the vicinity of $\beta_{t}$. For each $\beta$ above $\beta_{t}$, where flips from the $d$ to the $o$ phase may become frequent, we performed ten independent runs, each run being ten times shorter than at $\beta_{t}$. Flips, seen as jumps of the lattice average energy density $\bar{E}_{L}$, do occur, but the time histories show that the configurations with $\bar{E}_{L}$ larger than $\sim-1.24$ can be safely labelled as disordered. Although an accurate determination of $E^{*}$ from the data is not possible, we consistently find that the distribution $d_{\beta, A}(E)$ is essentially a straight line around $E=-1.1$, and we measure its slope (63) there. Our numerical results as a function of $\beta$ are reported in Fig.[5], and compared with the prediction (63) with $\beta^{*}$ fixed by the model (65). The agreement is significant: the spinodal point location could be identified numerically as the value of $\beta$ where data points for the left hand side of (63) linearly extrapolate to 0 .

We do not repeat the whole analysis for other lattice sizes. The important point is that the slope of $d_{\beta, A}(E)$ in the relevant energy region decreases as $L$ increases, as seen on Fig. [3]. Our discussion of Section (4.3) interpreted this size dependent behaviour of the energy distribution in terms of the singularity structure of the free energy. In particular, Eq. (56) indicates that, as $A \rightarrow \infty, d_{\beta, A}(E)$ as well as its derivative eventually goes to 0 , at least for any $E$ held fixed sufficiently far below the peak. Via Eq. (63), this in turn implies that $\beta^{*}(A) \rightarrow \beta_{t}$ when $A \rightarrow \infty$. Hence we conclude that the 
metastability interval $\beta^{*}-\beta_{t}$ shrinks to zero in the thermodynamical limit, reproducing the result of the Maxwell construction applied to Van der Waals theory.

\subsection{Free Energy for a Finite System in a Metastable State}

We have defined the disordered energy distribution up to $\beta^{*}$ as the distribution (59) cut below its minimum at $E_{m}$. It is then natural to use it in the standard relations (3, 4, 5) to associate a free energy $F_{A}(\beta)$ to the metastable disordered phase. So for $\beta \leq \beta^{*}$, we set

$$
\begin{aligned}
\frac{Z_{A}(\beta)}{Z_{A}\left(\beta_{t}\right)} & \equiv \exp \left(A\left(F_{A}(\beta)-F_{A}\left(\beta_{t}\right)\right)=\sum_{E}{ }^{\prime} D_{\beta, A}(E),\right. \\
\phi_{A}(z) & \equiv\left(\frac{f_{2}}{f}\right)^{3} \frac{1}{f_{2}}\left(F_{A}(\beta)-F\left(\beta_{t}\right)-f_{1}\left(\beta-\beta_{t}\right)\right) .
\end{aligned}
$$

In the above equations $\sum_{E}{ }^{\prime}$ means summation above $\max \left[E_{m},-2\right]$. Note the index $A$ in $F_{A}$ and $\phi_{A}$, to be explained soon. For consistency of course, one must find that, for $z \geq 0, \phi_{A}$ coincides with $\phi$, which by construction is $A$ independent. This can be checked by plugging in the representation (46) in the reweighted distribution (59), and the result in (66, 67), leading to

$$
\exp \left(A_{r} \phi_{A}(z)\right)=\frac{A_{r}}{2 \pi} \int_{\epsilon_{m}}^{\infty} d \epsilon \int_{-\infty}^{+\infty} d \rho \exp \left[A_{r}(\phi(\bar{z}+i \rho)-\epsilon(\bar{z}-z+i \rho))\right] .
$$

The lower bound $\epsilon_{m}$ corresponds to the cut $E_{m}$, when necessary. For $z \geq 0$, one may choose $\bar{z}=z, \epsilon_{m}$ can be set to $-\infty$ with an exponentially small error and integration over $\epsilon$ yields the desired result.

The choice $\bar{z}=z$ cannot be done for $z^{*}<z<0$, where $z^{*}$ corresponds to $\beta^{*}$ via (49), due to the branch point at $z=0$. Furthermore, the cut at $\epsilon_{m}$ becomes relevant. For both reasons, $\phi_{A}$ as defined by (67) actually becomes A-dependent. We illustrate this definition of the free energy for metastable states by the continuous line of Fig.[6], drawn for $L=120$ and $q=9$. Also shown is the result of the integral (67) with $\epsilon_{m}$ replaced by a fixed cut off (here corresponding to a cut at $E_{c u t}=-1.24$ ). For completness, we also plot the real part of the input $\phi(z)$ (37) for $z<0$ (dashed line). All definitions provide the same result for $z \geq 0$.

Let us add a few comments. First one observes that $\phi_{A}$ and Real $\phi$ are quite similar just below $z=0$. We know that $\operatorname{Im} \phi$ is very small there, or equivalently, that the droplet expansion (14) can be safely troncated 
at the size $\ell_{c}$ where the term of order $\ell$ is minimum [4, 1, 36]. Near the end point $z^{*}$, one then notice the intriguing fact that $\phi_{A}$ is not convex. However, because the restriction on the sum (66) depends on $\beta$ (via $E_{m}$ ), the energy cumulants do not coincide with the successive derivatives of $F_{A}(\beta)$, they must be computed by actual averages over the distribution (59). In particular, the quadratic fluctuations of the energy, of course always positive, are not proportional to $\phi_{A}^{\prime \prime}$, which thus may be negative.

Likewise, the fluctuation-dissipation theorem does not hold and the specific heat, $\left.C=-\beta^{2} d / d \beta<E\right\rangle$ is proportional neither to the quadratic fluctuations of the energy, nor to the second derivative of $\phi_{A}$. Averaging the energy, we compute $\langle E\rangle$ and find it has a minimum near $\beta^{*}$, showing that $C$ (after a maximum) does become negative. Such a phenomenon disappears in the thermodynamical limit since, in this limit, $\beta^{*} \rightarrow \beta_{t}$. If a fixed cut is set (dots in Fig. (5)), one recovers the familiar connection between energy cumulants and derivatives of the free energy, and $\phi$ is convex. Our choice of cutting at $E_{m}$ looks physically sensible. On a finite lattice, it leads to a situation similar to that of Van der Waals theory. In the thermodynamical limit, it defines a disordered microcanonical entropy density $S^{(d)}(E)$, defined and concave for $E^{(d)} \leq E \leq 0$. A similar construction of its ordered counterpart $S^{(o)}(E)$ is immediate through duality for $-2 \leq E \leq E^{(o)}$. The entropy $S(E)$ for the whole system is finally achieved as usual by supplementing $S^{(o)}(E)$ and $S^{(d)}(E)$ by their common tangent straight line between $E^{(o)}$ and $E^{(d)}$. In this way, our approach provides a model entropy $S(E)$ for any $E$.

\section{Summary and Conclusions}

We have applied a Fisher's version of the droplet picture to the $2 D q$-states Potts model in the vicinity of the temperature driven transition, which is first order above $q_{c}=4$. The droplet parameters were constrained by requiring that critical properties of the free energy are recovered as $q \rightarrow q_{c}$, complying with Fisher's scaling ideas and previous empirical observations based on large $q$ expansions of the energy cumulants [19, 20, 21]. Using duality of the Potts model, we focussed on the disordered phase properties and showed that, at the price of a unique $q$-independent free scale, the energy cumulants of order $\leq 10$ measured in a numerical simulation by Janke and Kappler were accurately reproduced (Fig.[1]). The analytical structure of the thermodynamical free energy in the inverse temperature $\beta$ plane was then analyzed, and a branch point essential singularity at the transition 
fully characterized. Next we investigated the consequences of these properties directly on the energy distribution currently generated in numerical simulations on finite lattices. In particular, evaluating this distribution analytically far away from its peak, we established a direct link between its size dependence there and the free energy discontinuity around the branch cut. Finite size effects are also present close to the peak of the distribution and in good agreement with the result of a numerical simulation which we performed at $q=9$ for comparison. We finally discussed static aspects of metastability. We defined a free energy beyond the transition point $\beta_{t}$ via the reweighting of the energy distribution at finite size, showing that, again due to the essential singularity, this can be done up to a size dependent spinodal point only, which moreover coincides with $\beta_{t}$ in the thermodynamical limit.

We noted earlier that various versions of the generic droplet picture have been used to study the first order transition of the Ising model below its critical temperature, and the associated issues of singularity of the free energy, metastability, and nucleation rates. As already mentioned in Section (2.3), the Fisher's version which we apply as been questionned for the Ising case in that it assigns a droplet an interfacial energy which grows faster than the perimeter $(\sigma>1 / 2$ in (14) $)$, which sounds odd at least for large droplets. Binder has shown [5, 9] how scaling prescriptions valid close to the critical point ( $T=T_{c}, h=0$ for Ising) can reconcile the Fisher's description and the original geometric picture of nucleation. We expect that similar arguments apply to the Potts case around $q=q_{c}, \beta=\beta_{t}$. In any case, our work shows that following the Fisher's point of view provides an extremely efficient and economical parametrization of the pure phase free energies, as particularly illustrated by its adequacy to describe the ten first energy cumulants: a) their measurements in numerical simulations [17] require the predicted, non-geometrical, values $\sigma=2 / 3$ and $\tau=7 / 3$ (see Eqs.(31, 32)), b) the pseudo interfacial tension $\omega$ scales as prescribed by the Widom exponent for the conventional tension.

We see three directions in which it would be interesting to pursue with the Potts model, noting that the above indices differ from their geometrical values more than they do for the Ising case. i) Derivation of exact results, in analogy with what has been done for Ising or Ising-like models on cluster distributions, large order cumulants, metastable states. ii) Dynamics at and near the transition and relationship between nucleation rates and singularities of the free energies. iii) Relationship between finite size properties of energy distributions at the transition and the discontinuity of the pure 
phase free energy. The existence of such a connection is demonstrated by our derivation of Eq. (56) (which can be easily extended to arbitrary values of the indices $\sigma$ and $\tau$ ). Accordingly, high statistics numerical data [17, 18] may provide a direct access to the free energy discontinuities, without requiring heavy dynamical investigations.

\section{Acknowledgments}

It is a pleasure to thank R.Balian, A.Billoire, T.Garel, W.Janke and R.Lacaze for their interest in this work and stimulating discussions.

\section{References}

[1] S.N. Isakov, Commun. Math. Phys. 95 (1984) 427.

[2] M.E.Fisher, Physics (N.Y.) 3 (1967) 255; in Critical Phenomena, Proc. of the Int. School of Physics "Enrico Fermi", Course LI, ed. M.S.Green, (Academic Press,1971) 1.

[3] J.E. Mayer and M.G. Mayer, Statistical Mechanics, Chapter 14, John Wiley, New York (1940).

[4] J.S.Langer, Annals of Physics 41 (1967) 108.

[5] K.Binder, Ann. Phys. 98 (1976) 390.

[6] F.Delyon,J. Stat. Phys. 21 (1979) 727.

[7] M.Aizenmann, F.Delyon and B.Souillard, J. Stat. Phys. 23 (1980) 267.

[8] J.D.Gunton, M.San Miguel and P.S.Sahni, The Dynamics of First-order Phase Transitions, in Phase Transitions and Critical Phenomena, Eds. C.Domb and J.L.Lebowitz, Vol. 8 (Academic Press, 1983).

[9] K.Binder, Rep. Prog. Phys. 50 (1987) 783.

[10] L.S.Schulman, in Ed. V.Privman Finite Size Scaling and Numerical Simulation of Statistical Systems, World Scientific, Singapore, 1990, p. 489 , and references therein.

[11] Dynamics of First Order Transitions, HLRZ, KFA Jülich Workshop, Ed. H.J.Herrmann, W.Janke and F.Karsch (World Scientific, Singapore, 1992). 
[12] D.B.Abraham and P.J.Upton, Int. Journal of Mod. Phys. C3 (1992) 1071.

[13] P.A.Rikvold and B.M.Gorman, Ed. D.Stauffer, Annual Review of Computational Physics, Vol. I, World Scientific, Singapore, 1994.

[14] C.C.A.Guenther, P.A.Rikvold and M.A.Novotny, Physica A 212 (1994) 194.

[15] Recent studies of nucleation include: V.Cataudella, G.Franzese, M.Nicodemi, A.Scala and C.Coniglio, Phys. Rev. E 54 (1996) 175; M.Acharyya and D.Stauffer, Eur. Phys. J. B5 (1998) 571; C.S.Schioppa, F.Sciortino and P.Tartaglia, Phys. Rev. E 57 (1998) 3797; L.Bocquet, F.Restagno and T.Biben, cond-mat/9901180. For a field theoretic approach: J.Alexandre, V.Branchina and J.Polonyi, Phys. Letters B 445 (1998) 351; A.Strumia, N.Tetradis and C.Wetterich, hep-ph/9808263.

[16] R. B. Potts, Proc. Camb. Phil. Soc. 48 (1952) 106.

[17] W.Janke and S.Kappler, J. Phys. (France) I7(1997) 663.

[18] A. Billoire, T. Neuhaus and B.A. Berg Nucl. Phys. 413 (1994) 795. This paper contains references to earlier attempts at interface tension determinations.

[19] T. Bhattacharya, R. Lacaze and A. Morel, Europhys. Lett. 23 (1993) 547; Nucl. Phys. B (Proc. Suppl.) 34 (1994) 671.

[20] T. Bhattacharya, R. Lacaze and A. Morel, J.Phys. (France) I7 (1997) 81.

[21] T. Bhattacharya, R. Lacaze and A. Morel, Nucl. Phys. B435 (1995) 526.

[22] J.Cardy, Renormalisation Group Theory of Branching Potts Interfaces, cond-mat/9806098.

[23] F.Y. Wu, Rev. Mod. Phys. 54 (1982) 235.

[24] R.J. Baxter, J. Phys. C6 (1973) L-445; J. Stat. Phys. 9 (1973) 145.

[25] B.J. Hiley and M.F.Sykes, J. Chem. Phys. 34 (1961) 1531. 
[26] J.W.Essam and M.E. Fisher, J. Chem. Phys. 38 (1963) 802; M.E.Fisher and M.F. Sykes, Phys. Rev. 114 (1974) 45; N.J.Guenther, D.A.Nicole and D.J.Wallace, J. Phys. A 13 (1980) 1755.

[27] P. W. Kasteleyn and C. M. Fortuin, J. Phys. Soc. Japan 26 (Suppl.), 11 (1969).

[28] H.Arisue and K.Kabata, The large $q$ expansion of the energy cumulants for the two-dimensional $q$-state Potts model, hep-lat/9810029.

[29] A. Klümper, A. Schadschneider and J. Zittarz, Z. Phys. B76 (1989) 247; A. Klümper, Int. Journal of Mod. Phys. B4 (1990) 871; E. Buffenoir and S. Wallon, J.Phys. A26 (1993) 3045.

[30] C. Borgs and W. Janke, J. Phys. (France) I2 (1992) 2011; W.Janke and S.Kappler, Nucl. Phys.(Proc. Suppl.) B42 (1995) 770; Europhys. Lett. 31 (1995) 345.

[31] B. Widom, J. Chem. Phys. 43 (1965) 3892.

[32] The function $\phi$ " can also be calculated as a Meijer's function (H.Navelet, private communication). Similar functions are studied in [33].

[33] H.A.Kastrup, cond-mat/9803269.

[34] C.Borgs and R.Kotecký, J. Stat. Phys. 61 (1990) 79; C.Borgs, R.Kotecký and S.Miracle-Sole, J. Stat. Phys. 62 (1991) 529.

[35] J.S.Langer Ann. Phys. 54(1969) 258.

[36] O.Penrose, J. Stat. Phys.78 (1995) 267.

[37] R.H.Schonmann and S.B.Shlosman, Comm. Math. Phys. 194 (1998) 389.

[38] S.B.Shlosman, in Statphys20, Eds. A.Gervois et al., Elsevier (North Holland) 1999. 


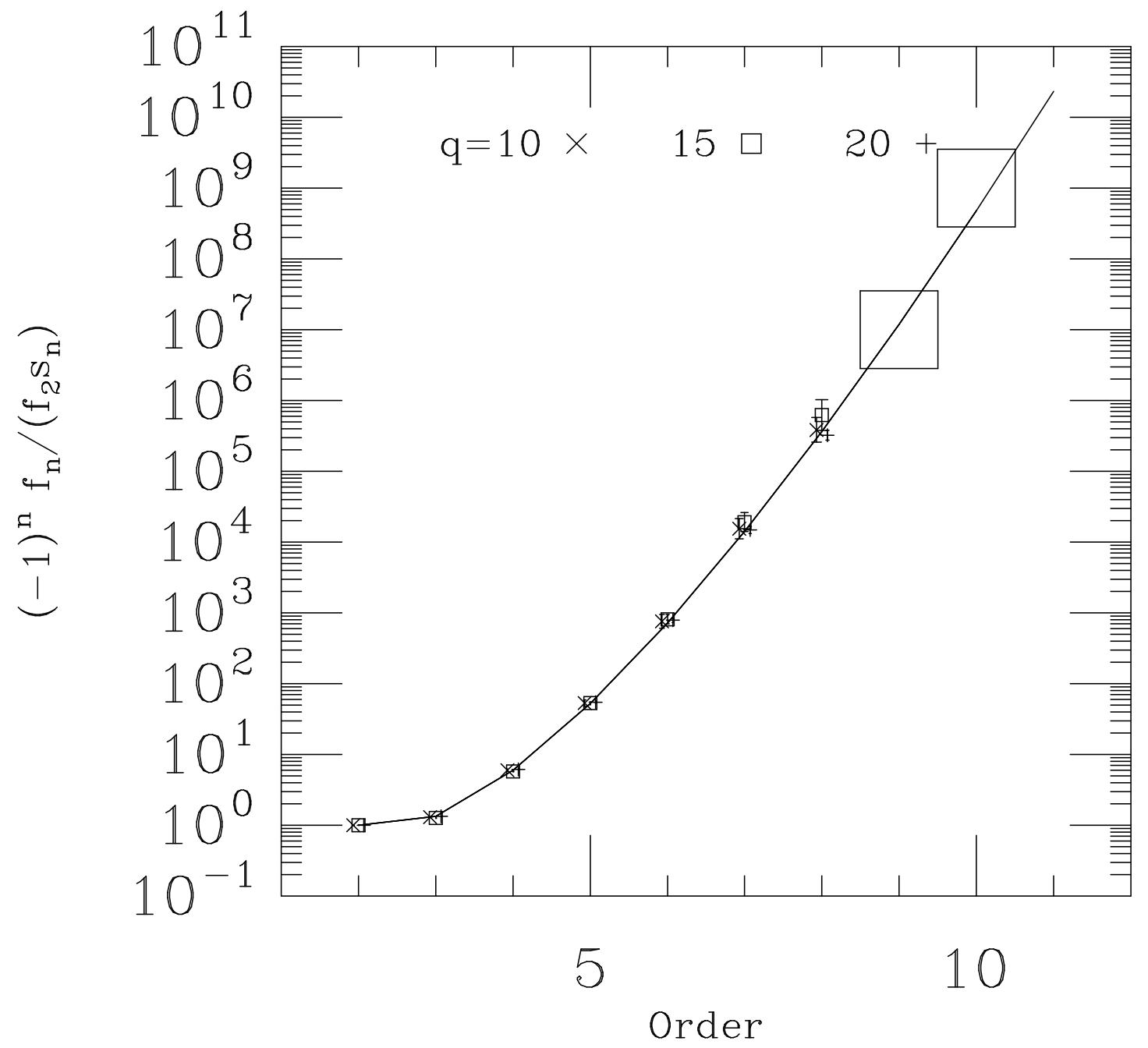

Figure 1: Comparison of the prediction of Eq.(28) (continuous line) with the numerical data of Ref. [17], versus the order $n$. The large squared symbols at $n=9$ and 10 indicate orders of magnitude only. The scale factor $S_{n}$, which contains the only free parameter $f$, exhausts the full $q$-dependence of the $f_{n}$ 's. 


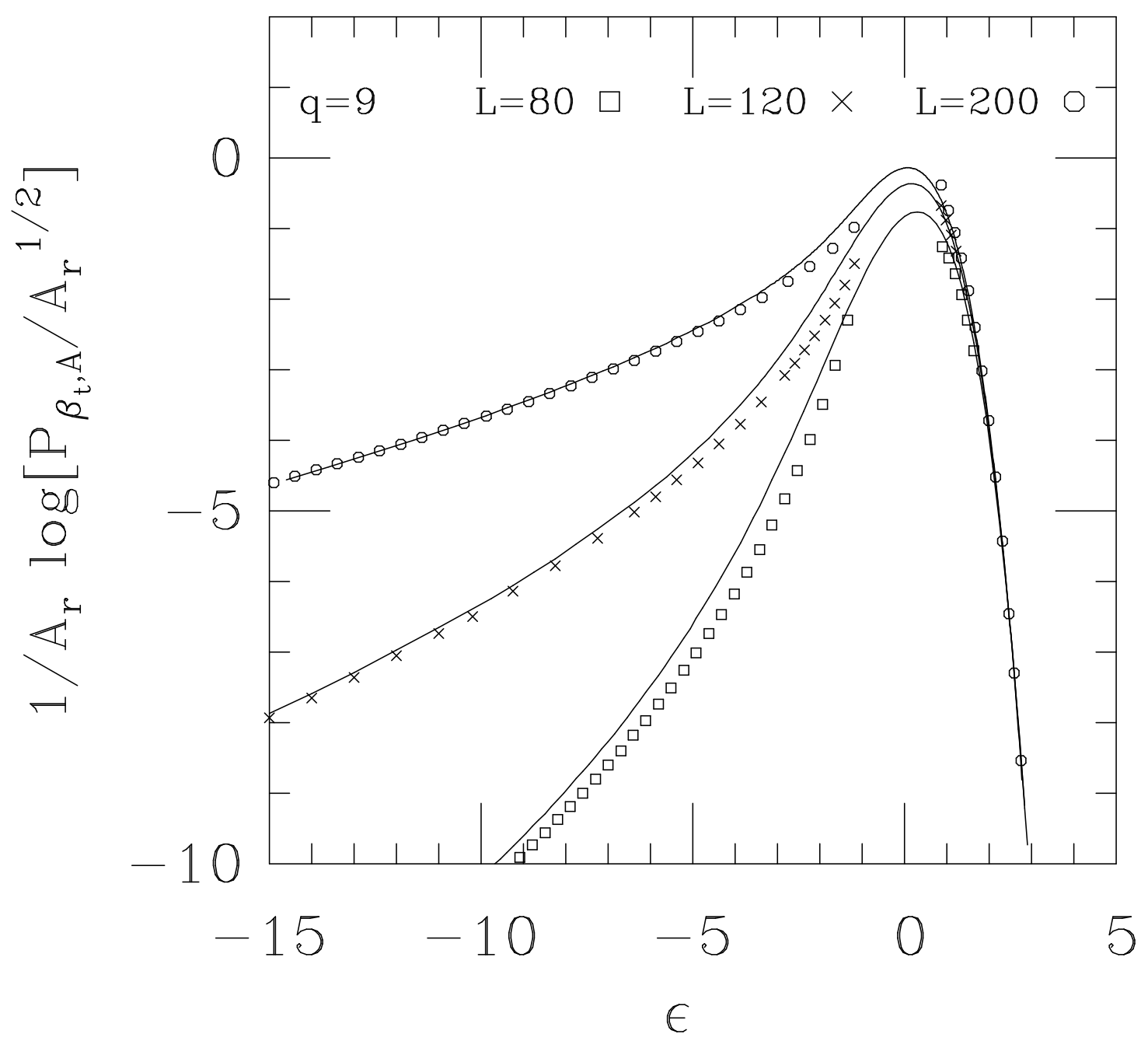

Figure 2: The energy distribution of the disordered phase at $q=9$ as a function of $\epsilon$ for $L=80,120,200$. The continuous curves result from numerical integration of (46) and the symbols represent analytical approximations (see text). As $L$ increases, the strong broadening for $\epsilon<0$ is a manifestation of the free energy singularity at $\beta_{t}$. 


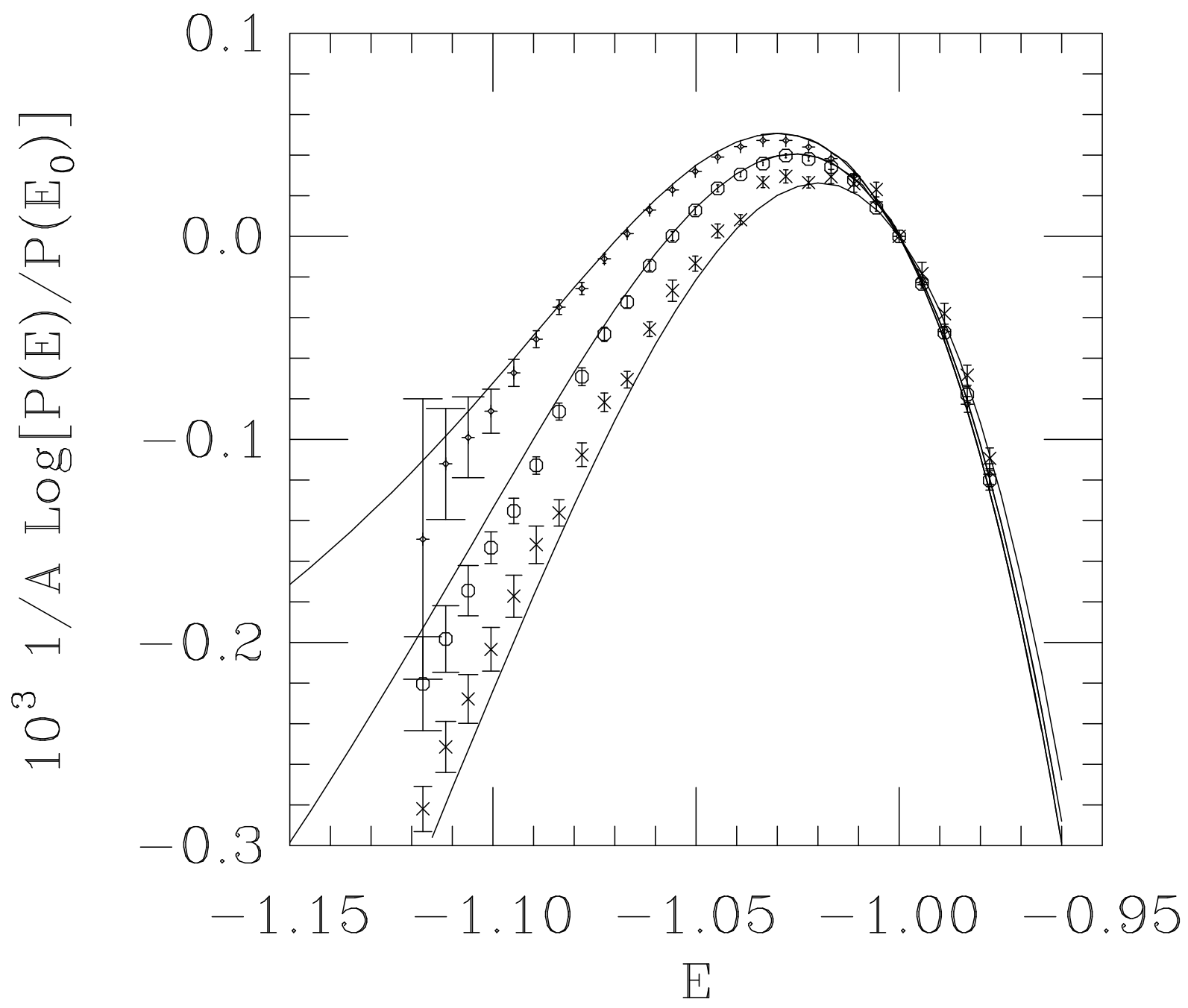

Figure 3: Comparison, for $p_{\beta_{t}, A}(E)$, Eq.(57), between the model predictions and numerical data taken at $q=9, L=80,120$, and 200 (from bottom to top). 


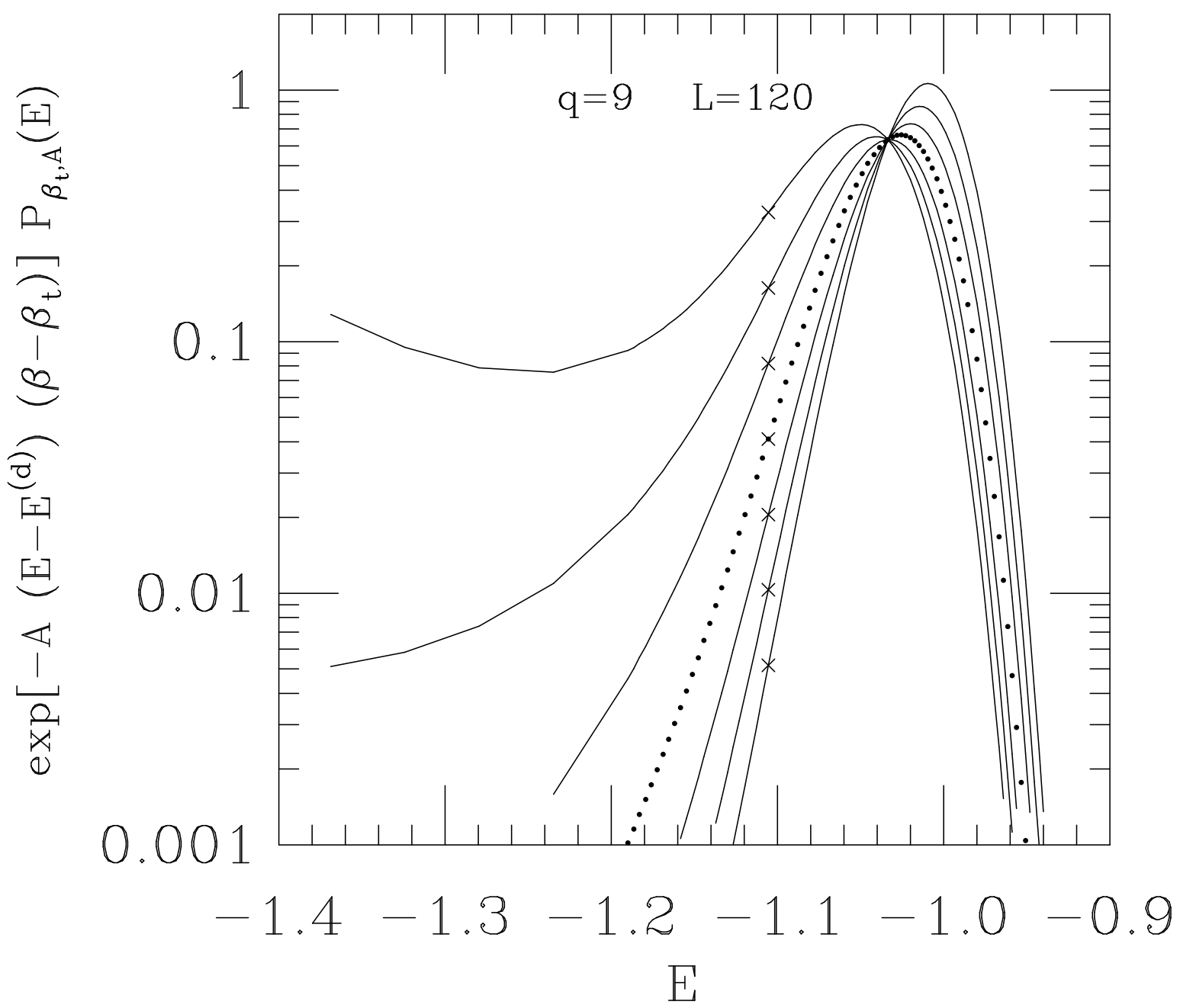

Figure 4: For the disordered phase at $q=9, L=120$, energy distributions for $\left(\beta-\beta_{t}\right)$ varying from $-210^{-3}$ to $210^{-3}$. On the left of the figure, temperature decreases from the bottom to the top curves (overcooling), the dotted curve corresponding to $\beta=\beta_{t}$. The crosses correspond to the inflexion points which all occur at the same $E^{*}=-1.104$. As the temperature is lowered below the transition, a minimum of the distribution enters the graph from the left. 


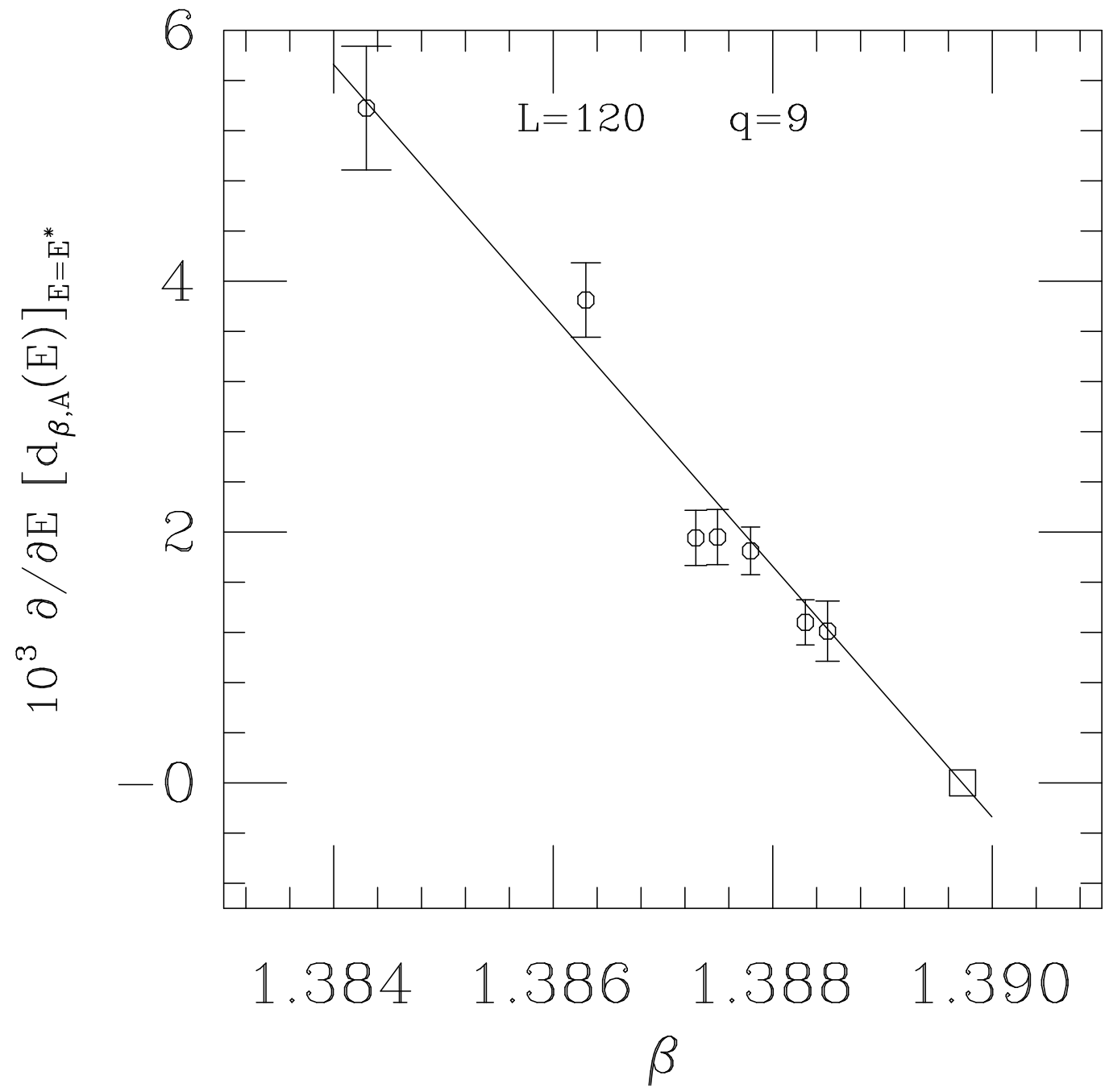

Figure 5: The slope $\frac{\partial}{\partial E} d_{\beta, A}(E)_{\left.\right|_{E=E^{*}}}$ as a function of $\beta$ from numerical simulations (o). The straight line represents Eq. (63), with $\beta^{*}$ given by the model (65): the slope vanishes at the spinodal value $\beta^{*}(\square)$. 


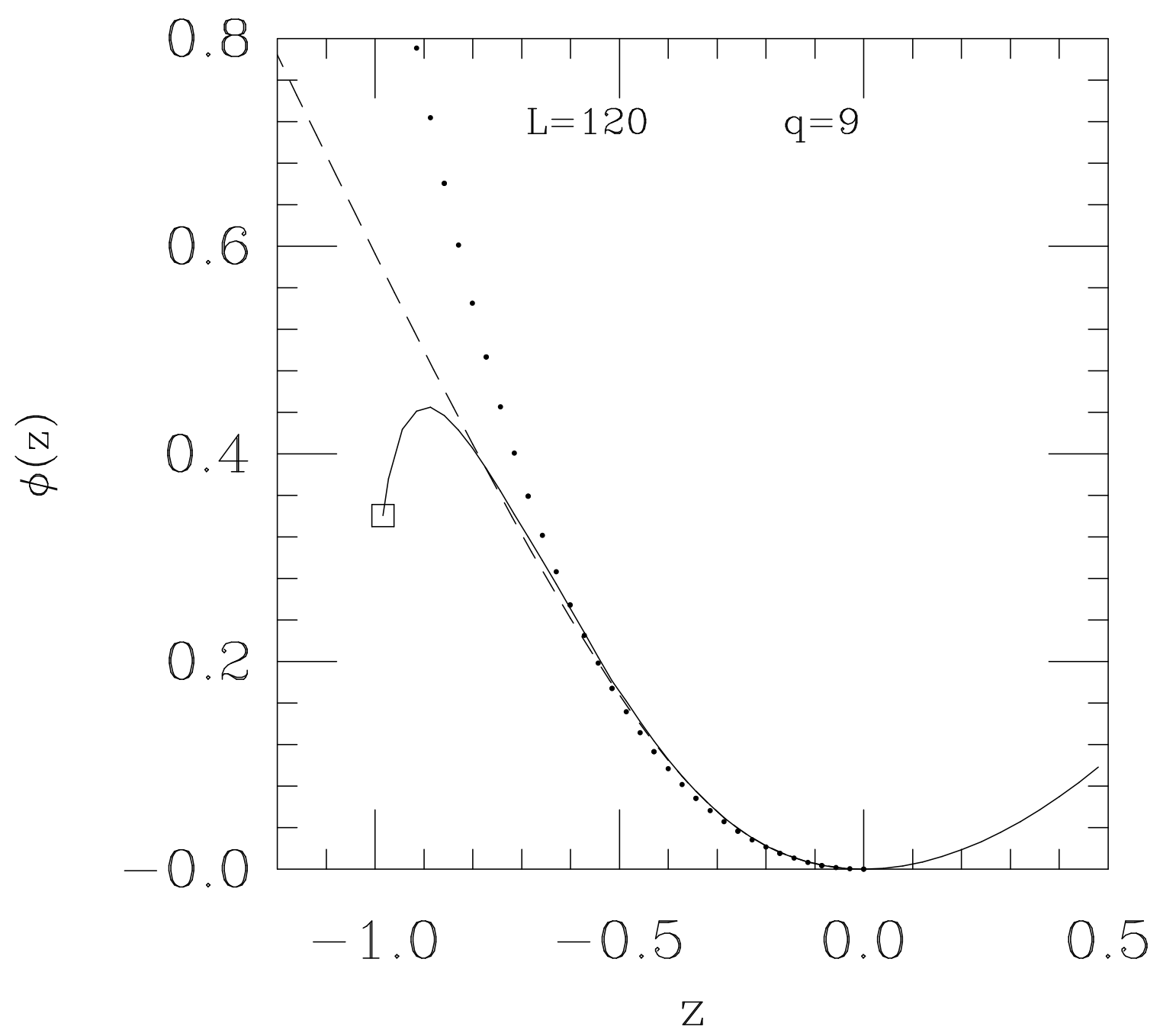

Figure 6: The rescaled free energy for metastable states $(z \leq 0)$. Are shown the sum (67) where the cut $\epsilon_{m}$ is the $z$ dependent minimum of the reweighted distribution (continuous curve), the same sum with a fixed cut $\left(E_{c u t}=-1.24\right.$, dots), and the real part of the input $\phi(z)$ (dashed curve). The symbol $\square$ marks the end point of the metastability region. It moves right and down with increasing $L$, ending at the origin in the thermodynamical limit. All definitions give the same answer $\phi(z)$ for $z \geq 0$. 